\title{
Lack of the metabotropic glutamate receptor subtype 7 selectively modulates Theta rhythm and working memory
}

\author{
Christian Hölscher, ${ }^{1,4,5}$ Susanne Schmid, ${ }^{2}$ Peter K.D. Pilz, ${ }^{2}$ Gilles Sansig, ${ }^{3}$ \\ Herman van der Putten, ${ }^{3}$ and Claudia F. Plappert ${ }^{2}$ \\ ${ }^{1}$ Department of Cognitive Neuroscience, University of Tübingen, Auf der Morgenstelle 28, Germany; ${ }^{2}$ Zoologisches Institut, \\ University of Tübingen, Auf der Morgenstelle 28, 72076 Germany; ${ }^{3}$ Novartis Institutes for BioMedical Research, Neuroscience \\ Research, CH 4002 Basel, Switzerland
}

\begin{abstract}
Metabotropic glutamate receptors (mGluRs) are known to play a role in synaptic plasticity and learning. We have previously shown that mGluR7 deletion in mice produces a selective working memory (WM) impairment, while other types of memory such as reference memory remain unaffected. Since WM has been associated with Theta activity (6-12 Hz) in EEGs, and since EEG abnormalities have been observed in these mice before, we studied the effect of mGluR7 gene ablation on EEG activity in the hippocampus, in particular in the Theta range, during performance of a WM task. In an eight-arm maze with four arms baited, mGluR7 knock-out (KO) and wild-type mice committed the same number of reference memory errors, whereas KOs committed more WM errors. While performing the task, $\mathrm{KO}$ mice showed substantially higher Theta amplitudes, and the ratio of Theta to overall EEG power was much increased. No change was seen in the Delta $(0-5 \mathrm{~Hz})$, or Gamma $(30-40 \mathrm{~Hz})$ EEG bands compared with controls. When recording EEGs during periods of rest in the home cages, no difference was seen between groups. These findings suggest that mGluR7 is important for modulation and control of Theta activity. Since only WM was affected, and only the Theta range of EEG activity was altered, these results show a correlation between Theta rhythm and WM performance, and therefore support the concept that Theta activity in the hippocampus is involved in WM storage.
\end{abstract}

Metabotropic glutamate receptors (mGluRs) are a family of glutamate-sensitive receptors that are coupled to intracellular signal transduction via G-proteins (Pin and Duvoisin 1995). It is known that mGluRs play an important role in the regulation of CNS synaptic plasticity, and also in some processes of memory formation. Pharmacological blockade of mGluR group III receptors (subtypes 4, 6, 7, and 8) has profound effects on the induction of synaptic plasticity (Bashir et al. 1993; Richter-Levin et al. 1994; Hölscher et al. 1997a), synaptic depression (Manahan-Vaughan 2000; Weber et al. 2002), and spatial learning (Hölscher et al. 1996, 1999).

In a previous study, we tested the effect of mGluR7 deletion on different forms of learning and memory formation (Hölscher 2003). We showed that mGluR7-deficient (knock-out; KO) mice were not impaired in a non-associative habituation task or in performing reference memory tasks of different complexities (open field, T-maze, four- and eight-arm mazes). However, in tasks that require increased capacity of working memory (WM) performance, mGluR7 KO mice showed clear deficits.

WM contains task-related information required to develop plans of action and to guide behavior (Baddeley and Hitch 1974; Olton et al. 1979). In the present paper, we adhere to the definition of WM by Olton et al. (1979), who emphasized the importance of hippocampal activity for supporting WM. How WM is actually encoded and stored in the brain has been under inves-

\footnotetext{
${ }^{4}$ Present address: School of Biomedical Sciences, University of Ulster, Coleraine BT52 1SA, Northern Ireland.

${ }^{5}$ Corresponding author.

E-mail c.holscher@ulster.ac.uk; fax 44-28-70324375.

Article and publication are at http://www.learnmem.org/cgi/doi/10.1101/ Im.98305.
}

tigation for a long time. The occurrence of oscillations in the EEG of subjects that performed WM tasks had been observed. In particular, oscillations in the Theta band have been postulated to be involved in mechanisms that underlie cognitive processes. Theta rhythm is the oscillation of membrane potentials in neuronal populations in the $6-12-\mathrm{Hz}$ frequency that can be observed in the EEG of moving or sensory-stimulated rats and mice (Vanderwolf 1969; Fox et al. 1983; Green and Greenough 1986; Buzsaki 2002). Theta activity is an indicator of attention processes and cognitive activity across species and is observed in particular during the performance of complex tasks that require WM. Several studies have described correlations between Theta activity and WM performance in humans, primates, and rodents (Givens and Olton 1995; Kahana et al. 2001; Jensen et al. 2002; Lee et al. 2005). Furthermore, the hippocampus of the rat appears to play an important role in WM storage. Hippocampal lesions or pharmacological blockade of hippocampal activity produce impairments in WM, such as the ability of rodents to bridge short time-delays introduced in WM tasks (Hölscher 2003). Previous work has shown that reducing Theta amplitude by pharmacological means impairs performance in WM tasks, thereby demonstrating that Theta most likely plays a functional role in the WM storage system (Givens and Olton 1990, 1995). Other studies have shown that the learning of an eye blink classical conditioning task is much reduced when stimulation occurs in the absence of Theta, while stimulation only in the presence of Theta produced good learning results (Seager et al. 2002; Griffin et al. 2004).

At the cellular level, Theta oscillations modulate neuronal firing probability (Buzsaki 2002) and the induction of synaptic plasticity (Hölscher et al. 1997b; Orr et al. 2001). One theory suggests that WM is held in store by mechanisms that tempo- 
A

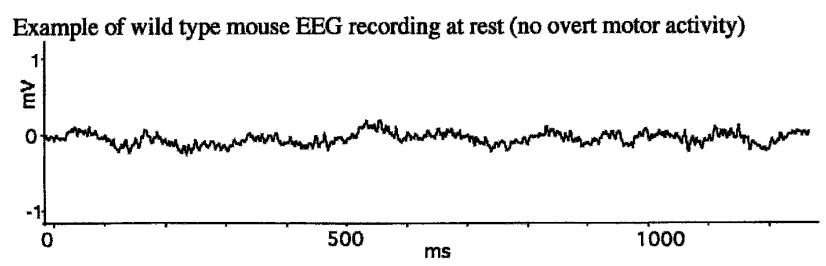

B

Fast -Fourier Transformation (FFT) of EEG

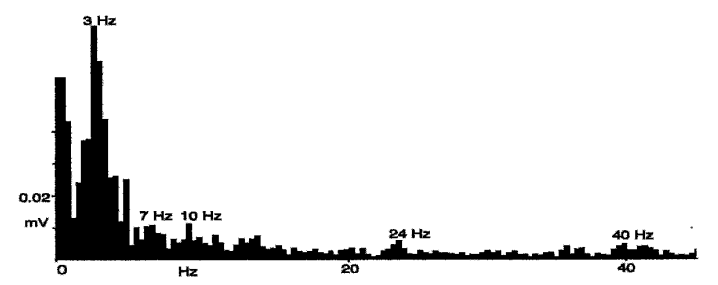

C

Example of mGluR7 KO mouse EEG recording at rest

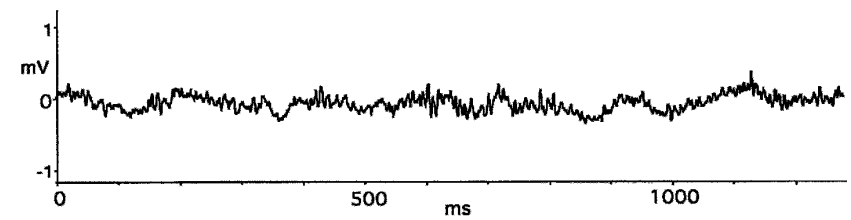

D

Fast-Fourier Transformation (FFT) of EEG

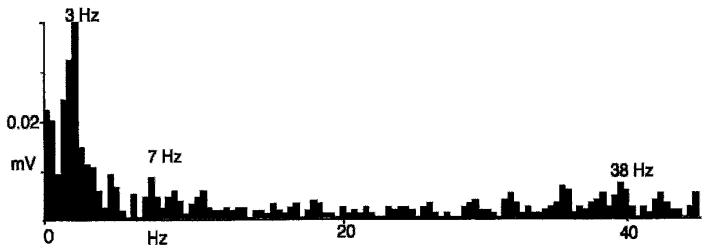

Figure 1. EEG recordings of mice at rest. $(A)$ A sample trace of a wildtype mouse is shown. (B) A fast-Fourier analysis of the power of the frequencies. Little Theta and Gamma activity is seen here; most activity is in the Delta band. (C) A sample recording of a mGluR7 KO mouse. (D) A fast-Fourier analysis of the power of the frequencies. Again, little Theta and Gamma activity is seen here; most activity is in the Delta band.

rarily induce synaptic plasticity for the duration of the task. It has been shown that electric stimulation of hippocampal neurons during Theta can affect synaptic transmission significantly. When stimulating hippocampal pathways in synchrony with Theta waves, long-term potentiation (LTP) of synaptic transmission can be induced on the peak of the wave, and long-term depression (LTD) on the trough of the wave (Hölscher et al. 1997b; Hyman et al. 2003). These mechanisms might be the basis for the synaptic changes that encode WM information during the performance of tasks.

To analyze the synaptic process involved in mGluR7 KO mice in more detail, we studied synaptic plasticity in hippocampal slices. Recordings from pyramidal cell-interneuron pairs revealed a delayed recovery from paired-pulse facilitation when stimulated at $10 \mathrm{~Hz}$. This shows that changes of the settings of inhibitory feedback loops in the Theta frequencies had occurred in these neuronal networks (Sansig et al. 2001; Bushell et al. 2002). We wondered whether Theta rhythm in these mice might be affected. These findings, together with the selective defects in WM observed in mGluR7 KO mice in the earlier study (Hölscher et al. 2004), prompted us to record hippocampal EEGs in mGluR7
KO during rest and during the performance of a complex WM task. Our hypothesis was that if Theta oscillations do play a role in supporting and storing WM, Theta rhythm should be affected in the mGluR7 KO mice.

\section{Results}

\section{EEG recordings}

The EEG was analyzed for activity in the three major frequency bands, Delta (0-5 Hz), Theta $(6-12 \mathrm{~Hz})$, and Gamma $(30-40 \mathrm{~Hz})$. During the rest condition in the home cage, EEG activity in the Delta band was high, and very little Theta or gamma was observed (Fig. 1). The recordings were conducted when the animal was at rest, with no visible motor activity, to avoid contamination by motor activity-induced Theta. Residual Theta was visible during exploration and sniffing, grooming, and other activities, even if the animal was not actually walking around. There was no difference between gender or between wild-type groups and $\mathrm{KO}$ groups under this condition (data not shown).

During the memory task, a higher activity level was observed in all groups in the Theta and Gamma band, while Delta activity was much reduced (Fig. 2). To maintain similar conditions during EEG recordings of the animals that were exploring

A Example of wild type mouse EEG recording during WM task

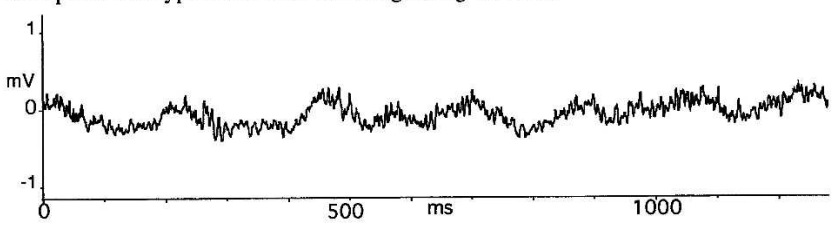

B

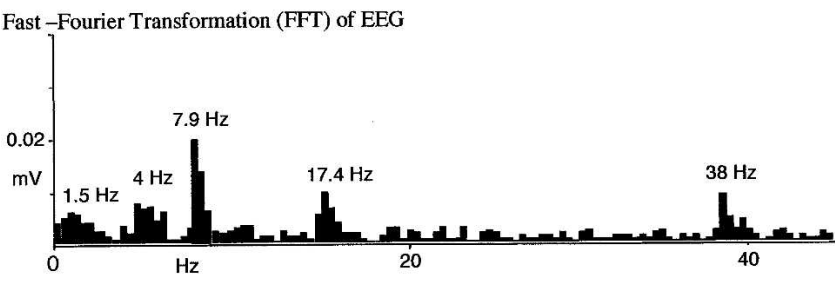

C

Example of mGluR7 KO mouse EEG recording during WM task

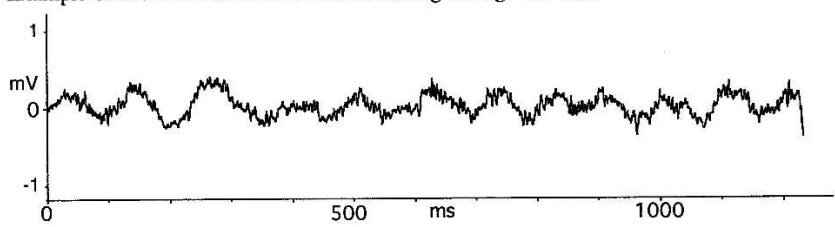

D

Fast-Fourier Transformation (FFT) of EEG

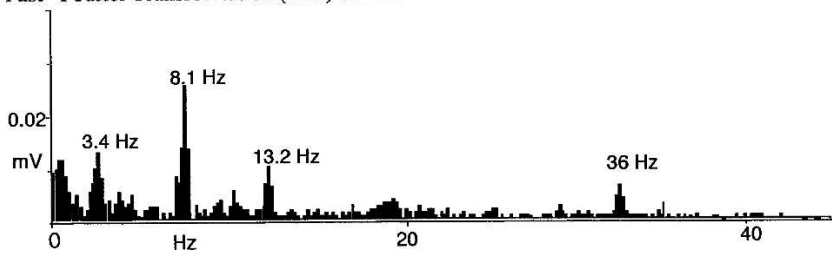

Figure 2. EEG recordings of mice during the performance of a WM task (while running along an arm). (A) A sample trace of a wild-type mouse is shown. (B) A fast-Fourier analysis of the power of the frequencies. Little Delta activity is seen here; most activity is in the Theta and Gamma band. (C) A sample recording of a mGluR7 KO mouse. (D) A fast-Fourier analysis of the power of the frequencies. Again, little Delta activity is seen here; most activity is in the Theta and Gamma band. 
the maze, only EEG recordings during running activity in an arm were analyzed. Since Theta is dependent on travel speed and motor activity, the conditions of motor activity had to be identical in all groups. EEG sections with contamination by jaw muscle movements during chewing were discarded. The animals were always active and moved from arm to arm to explore the maze; very few animals stopped moving during the trials or stayed in one arm for longer periods of time (behavior often seen in albino rats), so each recording session contains movementrelated Theta as well as any sensory/memory-related Theta. There was no gender effect (Fig. 3), so it was possible to pool the data to obtain two groups with 11 wild-type and $10 \mathrm{KO}$ animals. The main effect was observed during the WM task condition: A difference between Theta activity was found between $\mathrm{KO}$ and wildtype control groups. KO mice had substantially higher Theta amplitudes, and the ratio of Theta to overall EEG power was much increased (one-way ANOVA $p<0.0001$, post-hoc Bonferroni test ${ }^{* *}=p<0.01$ ). No change is seen in the other bands (Fig. 4).

\section{Eight-arm maze task, four arms baited}

There was a significant difference between $\mathrm{KO}$ and wild-type groups in the number of WM errors committed (two-way MANOVA, $\left.F_{(1,19)}=104.6, p<0.0001\right)$. KO animals repeatedly visited arms that they had already visited in that run. Wild-type animals rarely visited arms more than once, but did make some WM errors throughout the experiment. In a post-hoc NewmanKeuls multiple comparison test for each day, it was found that there was a persistent difference between numbers of WM errors committed when comparing $\mathrm{KO}$ and wild-type groups (days 1-5, 7, and 8: $p<0.01$; days 6 and 9: $p<0.05$ ). There was no difference in the number of LTM errors committed between the groups (Fig. 5). $\mathrm{KO}$ animals did reduce the number of visits to arms that had never been baited. The performance of $\mathrm{KO}$ animals was identical to that of wild-type animals; on no day was there a significant difference.

\section{Speed analysis}

Since locomotion has an impact on the onset and amplitude of Theta (Vanderwolf 1969; Czurko et al. 1999; for review, see Hölscher 2003), we analyzed the speed at which the animals traveled. In a previous study it was found that the number of line crossings in the open field per time unit was not significantly different (Hölscher et al. 2004). This showed that the KO animals were not overtly different in their excitability or basic motor activity compared with the wild-type group. In the present study, the number of arm visits and travel distance per time unit had

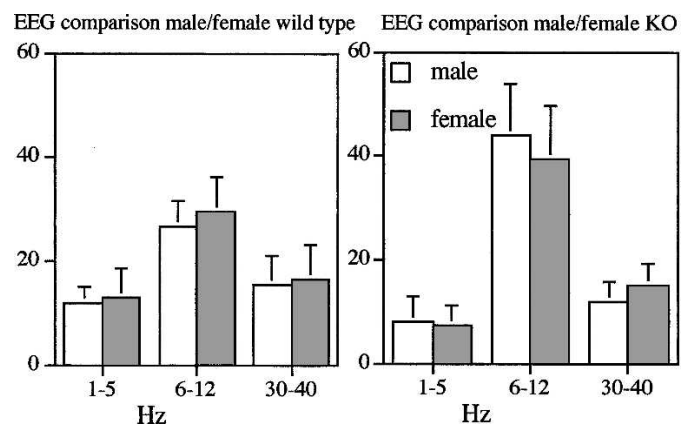

Figure 3. EEGs during performance of a WM task. Comparison of the EEG power in the three different frequency bands normalized for overall power of the EEG. The relative power is strongest in the Theta range during performance of the WM task, but there is no gender difference. Groups consisted of six male wild-type and five female wild-type animals, and of five male and five female KO animals.
EEG comparison KO vs control

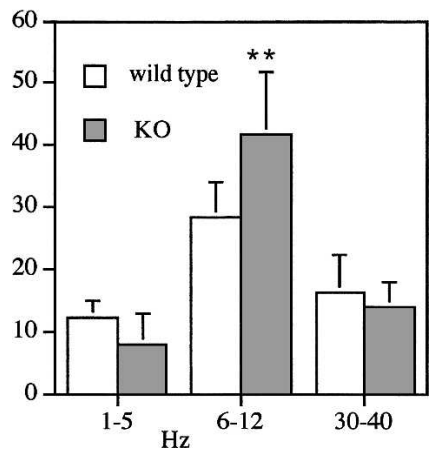

Figure 4. Comparison of the EEG power in the three different bands normalized for overall power of the EEG while performing a WM task. A difference in the relative power of Theta activity between wild-type and mGluR7 KO groups was observed (ANOVA $p<0.001, p<0.01$ ). No difference in the other frequency bands was seen. Groups consisted of 11 wild-type and $10 \mathrm{KO}$ animals.

been measured. The number of arm visits per trial was not significantly different between groups $(7.4 \pm 1.2$ SEM per 5 min in the KO group, and $6.8 \pm 2.2$ SEM in the wild-type group). Analysis of running speed of four wild-type mice and four KO mice showed no difference: wild-type $=57 \pm 5.4 \mathrm{SEM} \mathrm{cm} / \mathrm{sec}$; $\mathrm{KO}=68 \pm 8.7 \mathrm{SEM} \mathrm{cm} / \mathrm{sec}$. $T$-test $p>0.05$.

\section{Discussion}

The results of this study confirm the finding of our previous work (Hölscher et al. 2004), which demonstrated that mice lacking mGluR7 show selective performance deficits in tasks assessing WM, but no deficits in reference memory tasks. The EEG recordings during rest showed a high Delta band activity as expected in animals that are either inactive or in non-REM sleep (Vanderwolf 1969). Under these conditions, no differences were seen between wild-type and $\mathrm{KO}$ groups. As expected, during performance of the WM task, Theta and Gamma activity increased in all groups while Delta activity diminished (Vanderwolf 1969). Under these conditions, $\mathrm{KO}$ mice displayed a larger increase in Theta activity, suggesting that fine modulation of Theta activity is impaired in these mGluR7 KO mice. Interestingly, these changes in Theta amplitude were not accompanied by changes in the Gamma frequency band, suggesting that the latter oscillations are most likely not affected by the lack of mGluR7. The selective change in Theta power also suggests that the result cannot be due to differences in electrode location or recording techniques, which most likely would have affected all EEG frequencies in a similar way.

\section{How does the deletion of mGluR7 affect Theta?}

mGluR7s are selectively enriched at presynaptic sites of excitatory pyramidal cell terminals that project onto a subset of GABAergic interneurons in CA1 (Shigemoto et al. 1997). mGluR7s are also present on the presynaptic site of subsets of GABAergic interneurons (Somogyi et al. 2003), where they can reduce GABA release and alter local inhibition (Kogo et al. 2004). Our earlier electrophysiological findings showed that recovery from facilitation in paired stimuli given at $10 \mathrm{~Hz}$ was delayed in the cortex slice preparations from mGluR7 KO mice (Sansig et al. 2001). This reduced ability to decrease initial synaptic facilitation can explain the increased Theta amplitudes observed in the present study. While baseline inhibition was not altered (which would have greatly affected neurotransmission and EEG oscillations), the resetting mechanisms that reduce facilitation in the Theta 

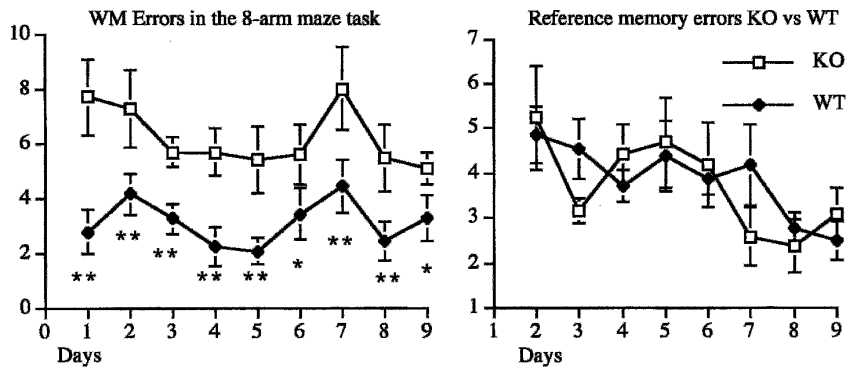

Figure 5. WM and reference memory errors in an eight-arm task, with four arms baited. The number of WM errors committed was higher in the KO group (two-way MANOVA $p<0.0001$ ). This deficit was visible until the last day $\left({ }^{*}=p<0.05 ;{ }^{* *}=p<0.01\right)$. No difference was observed in numbers of reference memory errors committed. Groups consisted of 11 wild-type and $10 \mathrm{KO}$ animals.

range were impaired, and this could have produced a persistent increase of neuronal excitability and oscillatiory amplitudes.

\section{How can increased Theta affect WM?}

Several studies have described correlations between Theta activity and WM performance (Givens and Olton 1995; Sauseng et al. 2004; Jensen and Lisman 2005; Lee et al. 2005). The reduction or block of Theta rhythm interferes with the performance in various WM tasks (Winson 1978; Givens and Olton 1990, 1995), and the amplitude of Theta correlated with the ability to perform well in WM tasks (Klimesch et al. 2001; Seager et al. 2002; Griffin et al. 2004). It appears counter-intuitive that higher amplitudes of Theta would reduce performance in WM tasks. However, it is important to keep in mind that the increase in amplitude observed in the present study is most likely due to a lack of modulation of local inhibition and a resetting of synaptic facilitation. In previous studies of WM performance and Theta amplitudes, the increase in amplitude was not caused by impaired inhibitory control but rather by a controlled increase in oscillatory network activity. The uncontrolled increase in Theta amplitudes could easily affect important network properties such as neuronal firing threshold and correct spike timing.

One theory proposes that Theta waves represent WM "storage units" that have space for four to seven spikes from individual neurons. The individual neurons are part of neuronal networks that are separated by gamma rhythm (Lisman and Idiart 1995). This resembles the limit of human WM storage capacity for random items, which is generally limited to four to seven items (Ebbinghaus 1885; Baddeley and Hitch 1974). Recordings in behaving animals indeed show that four to seven spikes usually occur within a Theta wave time window. Each spike can be produced by a separate neuron that in turn could be part of a separate active neuronal network, altogether encoding different contents (O'Keefe and Recce 1993; Harris et al. 2001). An increase in Theta amplitude could interfere with the correct timing of individual spikes and could affect WM storage by interfering with the correct order of items. Also, a more pronounced reduction of Theta inhibition would lead to an increase of firing probabilities in larger neuronal population, thereby reducing the "signal to noise" ratio. This would mean that the neurons carrying information fire along with randomly firing neurons that ordinarily would not have fired.

Another possibility is that the induction of synaptic plasticity by short spike trains (as observed in freely moving rats) is impaired. So-called "complex spike" trains of around five spikes are observed in the hippocampus of exploring rats ( $\mathrm{O}^{\prime}$ Keefe and Recce 1993). When imitating such spike train activity using electrical stimulation of hippocampal neurons during Theta, LTP can be easily induced. When stimulating with around five stimuli on the peak of Theta wave, LTP of synaptic transmission can be induced, and when stimulating at the trough of Theta waves, LTD or depotentiation of LTP can be observed (Hölscher et al. 1997b; Hyman et al. 2003). This mechanism might be the basis for synaptic changes that encode WM information during performance of tasks. By altering the amplitudes of Theta waves, the correct balance between LTP and LTD could be impaired, and thus WM storage would be greatly disturbed.

Our findings are consistent with models that propose a functional role for Theta amplitude in WM formation. Increased or decreased Theta amplitude, and an associated change in strength of local inhibition, should impact on neuronal firing probability and spike timing (Buzsaki 2002). If the Theta amplitude is too low, neurons might not reach depolarization thresholds and thus could not fire when required, or they may fire too late and the spike timing would then be off. If Theta amplitude is too high, depolarization of neurons would reach the firing threshold too quickly, and the time window in which an optimal excitation/inhibition balance normally is achieved to control pyramidal cell activity would become too short. Also, more neurons would be able to reach the firing threshold and fire, which would interfere with the signal transmission of neurons that carry information. Single-cell recordings in behaving mice are needed to address these questions further. In particular, it would be of interest to analyze whether the spike timing in relation to Gamma and Theta waves is affected in mGluR7 KO mice.

We conclude that the results presented here suggest that mGluR7 plays a role in the modulation of Theta network oscillations in the hippocampus and in the mechanisms that underlie or affect maintenance of working memory.

\section{Materials and Methods}

All animal experiments had been licensed by German licensing bodies according to German and EC law (BGBl. I S. 1105).

\section{Generation of knock-out mice}

The generation of mGluR7-deficient mice has been described elsewhere (Sansig et al. 2001). Part of the first coding exon comprising the $5^{\prime}$ untranslated region and the first 164 amino acids of mGluR7 was replaced with the neomycin-resistance gene by homologous recombination in E14 (129/Ola) embryonic stem (ES) cells. Aggregation with properly targeted ES cell clones yielded germ-line chimeras that were crossed with C57BL/6 mice for 15 generations. Homozygous mGluR7 $-/-$ and wild-type $(+/+)$ mice were generated by mating F15, B6-backcross generation mice heterozygous $(+/-)$ for the mGluR7 mutant gene, and their gender-matched littermate offspring aged $>12$ wk were used in the behavioral studies reported here. Animals were genotyped by PCR. All animals were trained in the different experiments in the order presented here.

\section{Housing}

Animals were housed individually in polyester cages with metal grid lids in temperature-regulated rooms $\left(21^{\circ} \mathrm{C}\right)$ with a 12 -h light / 12-h dark cycle. Food pellets and water were available ad lib. In experiments that involved food reward, animals were slightly food deprived for $2 \mathrm{~d}$ before the start of each experiment ( $4 \mathrm{~g}$ of food per animal/day). Food pellets used in the reward experiments were Choco Krispies (Kellogg's). Six wild-type males, five wild-type females, five $\mathrm{KO}$ males, and five $\mathrm{KO}$ females were used in this experiment.

\section{Surgery}

Mice were anaesthetized with $75 \mathrm{mg} / \mathrm{kg}$ Ketamin (WDT, Germany) $+5 \mathrm{mg} / \mathrm{kg}$ Xylazin (Rompun, Bayer) s.c. A local injection of lidocaine was given into the scalp, and the skin was cut and 
pulled back. Animals were mounted to a stereotaxic frame. A 0.8-mm hole was drilled into the skull and a stainless steel screw was implanted that acted as ground for the recording. Bipolar electrodes $(0.125 \mathrm{~mm}$ diameter, tungsten with Teflon coating; Bilaney) were implanted unilaterally $1.8 \mathrm{~mm}$ posterior and 1.2 $\mathrm{mm}$ lateral to the midline. These values had been obtained previously by injecting dye into a mouse brain. The electrodes were slowly lowered through the cortex and the upper layers of the hippocampus and into the CA1 region until the appearance of EEG Theta activity and the observation of sharp waves (O'Keefe and Recce 1993). Recordings of EEGs were made from the stratum radiatum in close proximity to the stratum lacunosummoleculare in the CA1 region of the right hippocampal hemisphere. The electrodes were fixed to the skull by dental cement that was anchored to the skull by stainless steel screws.

EEGs were recorded on a computerized stimulating and recording unit (PowerLab, ADI instruments). Sampling rate was at $1 \mathrm{kHz}$. Data were filtered above $1 \mathrm{Khz}$. All data were saved to disk for further analysis. Data were spectral analyzed by Fast Fourier analysis (Powerlab and Matlab software).

Locations of electrodes were analyzed after the experiment. The brains of the animals were removed, and a block including the hippocampus was cut and fixed in PBS-buffered formalin $(8 \%)$. Sections were cut on a vibratome, stained by hematoxylin and eosin, and examined by light microscopy.

\section{EEG recordings}

EEGs were recorded by attaching the recording wires to the electrodes via a connector (animals were briefly anaesthetized using Halothane) and letting the animals adapt to the wire. This connector also contained an LED that was used for the tracking system. Wires were suspended to the ceiling or the recording chamber via rubber bands to avoid entanglement. Animals did not display any stress or altered behavior caused by the wires.

\section{Recording at rest}

When recording EEGs at rest, animals were given 15 min to settle down in the home cage. A series of recordings over 10 min was made. Only EEG traces during periods of rest were analyzed, when the animal was not moving around or showing other overt motor activity such as exploring or sniffing. The episodes of rest were indicated by press of a button that conveyed a TTL signal to the recording system. The signal was recorded along with the EEG to indicate which sections were rest EEG recordings.

\section{Recording during WM performance}

Animals were made familiar with the room and with the maze. In pretraining sessions, all arms were baited to familiarize the animals with the task. During the performance of the task, animals were attached to the wires and left to explore the maze and to learn the location of baited arms. Each recording session lasted -5-10 min, after which the animal had completed the task. To keep the recording conditions consistent, the sections of EEG traces were analyzed that had been recorded when the animals were running along an arm. Since jaw muscle movements during chewing of pellets sometimes produced interference in the EEG, these sessions were discarded. Experimenters were blinded to the identity of the mice. The position of animals was recorded by a tracking system (AXONA) that analyzed the location of the mouse and stored it as $\mathrm{X}-\mathrm{Y}$ coordinate files (as measured by the LED on its head). The LED emitted light in the UV spectrum not visible to the mouse. The tracking of the animals allowed for the discrimination between episodes of activity and inactivity and location within the maze. Tracks were analyzed off-line, and distances per time were estimated by taking 10 segments lasting 3 min each for wild-type mice $(n=4)$ and comparing them with 10 segments taken from KO mouse recordings $(n=4)$. The travel speed was estimated for each group.

\section{Eight-arm (radial arm) maze}

The eight-arm maze was constructed from chip board with white PVC coating. The length of each arm was $68 \mathrm{~cm}$, width $17 \mathrm{~cm}$, and height $35 \mathrm{~cm}$. The diameter of the central platform was 50 $\mathrm{cm}$. At the end of each arm a square black plastic dish with $5 \mathrm{~cm}$ side length was attached.

Task

In this task, four out of eight arms were baited. The same arms were baited throughout the experiments. The aim of the task was to retrieve all the food pellets. Visits to arms that already had been visited in the run were considered working memory errors. After day one, visits to arms that were never baited were considered reference memory errors, which is a type of long-term memory error in this design since the animals had $9 \mathrm{~d}$ to learn which arms were baited. Each animal had two runs per day for 9 d (see also Hölscher et al. [2004] and Jarrard [1983] for more details on this task).

\section{Statistics}

The mean of values \pm SEM are presented. Significance is reported if $p<0.05$. Two-way repeated measures ANOVA or ANCOVA (Statistica) were used to analyze differences between groups, effects over time, and interactions. In order to investigate WM differences between days, a Newman-Keuls multiple comparison test was applied post hoc to the ANOVA analysis in some data sets. For speed analysis, an unpaired two-tailed $t$-test was used.

\section{References}

Baddeley, A.D. and Hitch, G.J. 1974. Working memory. Oxford University Press, New York.

Bashir, Z.I., Jane, D.E., Sunter, D.C., Watkins, J.C., and Collingridge, G.L. 1993. Metabotropic glutamate receptors contribute to the induction of long-term depression in the CA1 region of the hippocampus. Eur. J. Pharmacol. 239: 265-266.

Bushell, T., Sansig, G., Collett, V., van der Putten, H., and Collingridge, G. 2002. Altered short-term synaptic plasticity in mice lacking the metabotropic glutamate receptor mGlu7. Sci. World 2: 730-737.

Buzsaki, G. 2002. Theta oscillations in the hippocampus. Neuron 33: $325-340$.

Czurko, G., Hirase, H., Csicsvari, J., and Buzsaki, G. 1999. Sustained activation of hippocampal pyramidal cells by "space clamping" in a running wheel. Eur. J. Neurosci. 11: 344-352.

Ebbinghaus, H. 1885. Über das Gedächtnis. Duncker \& Humblot, Leipzig.

Fox, S.E., Wolfson, S., and Ranck, J.B. 1983. Investigating the mechanisms of hippocampal theta rhythms: Approaches and progress. In Neurobiology of the hippocampus (ed. W. Seifert), pp. 303-319. Academic Press, London.

Givens, B.S. and Olton, D.S. 1990. Cholinergic and GABAergic modulation of medial septal area: Effect on working memory. Behav. Neurosci. 104: 849-855.

. 1995. Bidirectional modulation of scopolamine-induced working memory impairments by muscarinic activation of the medial septal area. Neurobiol. Learn. Mem. 63: 269-276.

Green, E.J. and Greenough, W.T. 1986. Altered synaptic transmission in dentate gyrus of rats reared in complex environments: Evidence from hippocampal slices maintained in vitro. J. Neurophysiol. 55: 739-750.

Griffin, A.L., Asaka, Y., Darling, R.D., and Berry, S.D. 2004. Theta-contingent trial presentation accelerates learning rate and enhances hippocampal plasticity during trace eyeblink conditioning. Behav. Neurosci. 118: 403-411.

Harris, K.D., Hirase, H., Leinekugel, X., Henze, D.A., and Buzsaki, G. 2001. Temporal interaction between single spikes and complex spike bursts in hippocampal pyramidal cells. Neuron 32: 141-149.

Hölscher, C. 2003. Time, space, and hippocampal functions. Rev. Neurosci. 14: 253-284

Hölscher, C., McGlinchey, L., and Rowan, M.J. 1996. L-AP4 (L-(+)-2-amino-4-phosphonobutyric acid) induced impairment of spatial learning in the rat is antagonized by MAP4 ((S)-2-amino-2-methy 1-4-phosphonobutanoic acid). Behav. Brain Res. 81: $69-79$

Hölscher, C., Anwyl, R., and Rowan, M.J. 1997a. Activation of group II metabotropic glutamate receptors blocks induction of long-term potentiation and depotentiation in area CA1 of the rat in vivo. Eur. J. Pharmacol. 322: 155-163.

1997b. Stimulation on the positive phase of hippocampal theta rhythm induces long-term potentiation which can be depotentiated by stimulation on the negative phase in area CA1 in vivo. $J$. 
Neurosci. 17: 6470-6477.

Hölscher, C., Gigg, J., and O’Mara, S. 1999. Metabotropic glutamate receptor activation and blockade. Consequences for long-term potentiation, learning and neurotoxicity. Neurosci. Biobehav. Rev. 23: 399-410.

Hölscher, C., Schmid, S., Pilz, P., van der Putten, H., and Plappert, C. 2004. Metabotropic glutamate receptor subtype 7 ablation causes a selective deficit in working memory but not long-term memory formation. Behav. Brain Res. 154: 473-481.

Hyman, J.M., Wyble, B.P., Goyal, V., Rossi, C.A., and Hasselmo, M.E. 2003. Stimulation in hippocampal region CA1 in behaving rats yields long-term potentiation when delivered to the peak of theta and long-term depression when delivered to the trough. J. Neurosci. 23: $11725-11731$.

Jarrard, L.E. 1983. Selective hippocampal lesion and behavior: Effects of kainic acid lesions on performance of place and cue tasks. Behav. Neurosci. 97: 873-889.

Jensen, O. and Lisman, J.E. 2005. Hippocampal sequence-encoding driven by a cortical multi-item working memory buffer. Trends Neurosci. 28: 67-72.

Jensen, O., Gelfand, J., Kounios, J., and Lisman, J.E. 2002. Oscillations in the alpha band (9-12 Hz) increase with memory load during retention in a short-term memory task. Cereb. Cortex 12: 877-882.

Kahana, M.J., Seelig, D., and Madsen, J.R. 2001. Theta returns. Curr. Opin. Neurobiol. 11: 739-744.

Klimesch, W., Doppelmayr, M., Stadler, W., Pollhuber, D., Sauseng, P., and Rohm, D. 2001. Episodic retrieval is reflected by a process specific increase in human electroencephalographic theta activity. Neurosci. Lett. 302: 49-52.

Kogo, N., Dalezios, Y., Capogna, M., Ferraguti, F., Shigemoto, R., and Somogyi, P. 2004. Depression of GABAergic input to identified hippocampal neurons by group III metabotropic glutamate receptors in the rat. Eur. J. Neurosci. 19: 2727-2740.

Lee, H., Simpson, G.V., Logothetis, N.K., Rainer, G. 2005. Phase locking of single neuron activity to theta oscillations during working memory in monkey extrastriate visual cortex. Neuron 45: 147-156.

Lisman, J.E. and Idiart, M.A.P. 1995. Storage of 7 plus-minus 2 short-term memories in oscillatory subcycles. Science 267: 1512-1515.

Manahan-Vaughan, D. 2000. Group III metabotropic glutamate receptors modulate long-term depression in the hippocampal CA1 region of two rat strains in vivo. Neuropharmacology 39: 1952-1958.

O'Keefe, J. and Recce, M.L. 1993. Phase relationship between hippocampal place units and the EEG theta rhythm. Hippocampus
3: $317-330$.

Olton, D.S., Becker, J.T., and Handelmann, G.E. 1979. Hippocampus, space and memory. Behav. Brain Sci. 2: 313-365.

Orr, G., Rao, G., Houston, F.P., McNaughton, B.L., and Barnes, C.A. 2001. Hippocampal synaptic plasticity is modulated by theta rhythm in the fascia dentata of adult and aged freely behaving rats. Hippocampus 11: 647-654.

Pin, J.-P. and Duvoisin, R. 1995. The metabotropic glutamate receptors: Structure and functions. Neuropharmacology 34: 1-26.

Richter-Levin, G., Errington, M.L., Maegawa, H., and Bliss, T.V.P. 1994. Activation of metabotropic glutamate receptors is necessary for long-term potentiation in the dentate gyrus and for spatial learning. Neuropharmacology 33: 853-857.

Sansig, G., Bushell, T.J., Clarke, V.R., Rozov, A., Burnashev, N., Portet, C., Gasparini, F., Schmutz, M., Klebs, K., Shigemoto, R., et al. 2001. Increased seizure susceptibility in mice lacking metabotropic glutamate receptor 7. J. Neurosci. 21: 8734-8745.

Sauseng, P., Klimesch, W., Doppelmayr, M., Hanslmayr, S., Schabus, M., and Gruber, W.R. 2004. Theta coupling in the human electroencephalogram during a working memory task. Neurosci. Lett. 354: $123-126$.

Seager, M.A., Johnson, L.D., Chabot, E.S., Asaka, Y., and Berry, S.D. 2002. Oscillatory brain states and learning: Impact of hippocampal theta-contingent training. Proc. Natl. Acad. Sci. 99: 1616-1620.

Shigemoto, R., Kinoshita, A., Wada, E., Nomura, S., Ohishi, H., Takada, M., Flor, P.J., Neki, A., Abe, T., Nakanishi, S., et al. 1997. Differential presynaptic localization of metabotropic glutamate receptor subtypes in the rat hippocampus. J. Neurosci. 17: 7503-7522.

Somogyi, P., Dalezios, Y., Lujan, R., Roberts, J.D., Watanabe, M., and Shigemoto, R. 2003. High level of mGluR7 in the presynaptic active zones of select populations of GABAergic terminals innervating interneurons in the rat hippocampus. Eur. J. Neurosci. 17: 2503-2520.

Vanderwolf, C.H. 1969. Hippocampal electrical activity and voluntary movement in the rat. Electroenceph. Clin. Neurophysiol. 26: 407-418.

Weber, M., Schnitzler, H., and Schmid, S. 2002. Synaptic plasticity in the acoustic startle pathway: The neuronal basis for short-term habituation? Eur. J. Neurosci. 16: 1325-1332.

Winson, J. 1978. Loss of hippocampal theta rhythm results in spatial memory defects of the rat. Science 201: 160-163.

Received January 18, 2005; accepted in revised form August 11, 2005. 


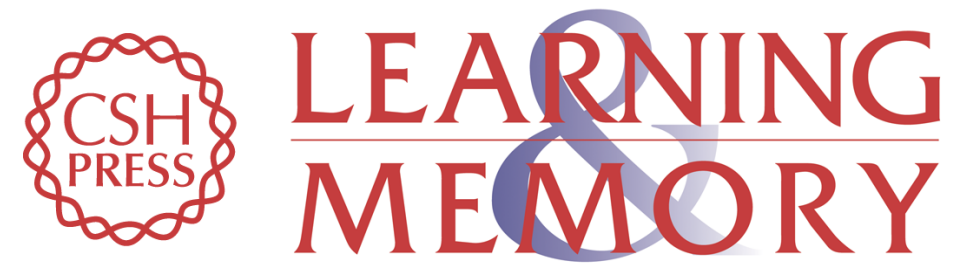

\section{Lack of the metabotropic glutamate receptor subtype 7 selectively modulates Theta rhythm and working memory}

Christian Hölscher, Susanne Schmid, Peter K.D. Pilz, et al.

Learn. Mem. 2005, 12:

Access the most recent version at doi:10.1101/lm.98305

References This article cites 38 articles, 7 of which can be accessed free at: http://learnmem.cshlp.org/content/12/5/450.full.html\#ref-list-1

License

Email Alerting

Receive free email alerts when new articles cite this article - sign up in the box at the Service top right corner of the article or click here. 